\title{
Optimal Allocation of Distributed Generation in Power Systems Considering Multiple Operative Scenarios
}

\author{
Jesús María López Lezama ${ }^{1}$, Nicolás Muñoz Galeano² and Fernando Villada Duque ${ }^{3}$ \\ ${ }^{I}$ Departamento de Ingeniería Eléctrica, Universidad de Antioquia Calle 70 No 52-21, Medellín 050010, Colombia. \\ ORCID: 0000-0002-2369-6173 (Jesús) \\ ${ }^{2}$ Departamento de Ingeniería Eléctrica, Universidad de Antioquia Calle 70 No 52-21, Medellín 050010, Colombia. \\ ORCID: 0000-0003-1407-5559 (Nicolás) \\ ${ }^{3}$ Departamento de Ingeniería Eléctrica, Universidad de Antioquia Calle 70 No 52-21, Medellín 050010, Colombia. \\ ORCID: 0000-0001-6577-2181 (Fernando)
}

\begin{abstract}
This paper presents a genetic algorithm (GA) approach to determine the optimal allocation of distributed generation (DG) in electric power systems considering multiple operative scenarios. Several indexes designed for the assessment of the network in terms of voltage and chargeability limits were defined to guide the metaheuristic algorithm. These indexes measure violations in chargeability and voltage limits for different operative scenarios. The objective function consists on minimizing the impacts of contingencies in the chargeability and voltage profile of power systems. To show the applicability and effectiveness of the proposed approach several tests were performed on the IEEE 30 and 57 bus tests systems. Results show that the proposed approach allows finding the allocation of DG that maximizes its positive impacts in terms of voltage profile and chargeability.
\end{abstract}

Keywords: Distributed generation, genetic algorithm, operative scenarios, power systems.

\section{INTRODUCTION}

Currently, there is a growing interest in distributed generation (DG) technologies driven by several factors that include a renewed ecological awareness, the cost reduction of smallscale generation technologies and new opportunities in the energy sector [1].

Several DG technologies are still in the development process, while others are mature and have reached commercial status such as photovoltaic and wind generation [2]. The technical and economic benefits of DG have been widely studied in the specialized literature. DG located in the distribution network can reduce power losses [3], improve the voltage profile of the network [4] and enhance network security [5]. Also, DG can defer investments [6] and boost the paradigm of smart grids [7].

The potential benefits that can be achieved through DG depend on its correct location and sizing. An incorrect location of DG can bring along several drawbacks such as increment of power losses, and problems with protection coordination. That is why many studies focus on finding and testing methodologies to optimize the location and sizing of DG both in distribution and transmission systems. This methodologies can be classified in classic mathematical programming and metaheuristic techniques [8]. The last ones are the most promising given their ability to deal with nonconvex and multimodal problems. Some metaheuristic techniques used for the optimal location and sizing of DG include genetic algorithms [8], particle swarm optimization [9], whale optimization [10] and immune algorithms [11]. A literature review regarding the location and sizing of DG can be consulted in [8].

A literature review on the topic of DG shows that most of the studies regarding its location and sizing are performed from the point of view of the distribution network. Nevertheless, this paper analyzes the impacts of DG from the standpoint of the transmission network. For this, it is assumed that the system planner desires to identify the best locations for DG in order to harvest its benefits. In this case, we have considered the security of the system which is measured in terms of postcontingency power flows and voltage limits violations. For this purpose, several indices were defined considering the operation of the network in normal conditions and under single contingencies. This is done for different operative scenarios.

From the point of view of the transmission network, the aggregation of DG units in a given bus can be seen as a reduction of the net demand of that bus. This reduction is variable depending on the technology of the DG units. For simplicity, the reduction of demand has been considered constant taking into account the net capacity factor defined as the ratio of the actual electrical energy output over a given period of time to the maximum possible electrical energy output over the same period. Four indexes are defined to identify the nodes in which the DG can bring more benefits, and a list of candidate nodes to allocate DG units is created. The DG assignment in the candidate nodes is done through a genetic algorithm. Several tests were performed with the IEEE 
30 and 57 bus tests systems showing the applicability and effectiveness of the proposed approach.

The rest of the document is divided as follows: Section 2 defines the proposed indexes and provides an outline of the selected metaheuristic. Section 3 presents the tests and results of the optimal allocation of DG considering two benchmark IEEE power systems; finally, conclusions are presented in Section 4.

\section{METHODOLOGY}

Four indexes, adapted from [12], were proposed to assess the performance of the transmission network in terms of violation of chargeability limits and voltage profile. In this case, we have considered normal operation and single contingencies (N-1 criterion) and several operative scenarios. The indexes are used as the fitness function of the PSO metaheuristic to find the best locations for DG. The operative scenarios are created taking into account that within the dynamic of an energy market, not all centralized units are always dispatched. Therefore, one centralized unit is removed at a time and a new dispatch is obtained dividing its initial generation equally among the remaining centralized generators. In consequence, there will be as many generation scenarios as centralized generators.

\subsection{Proposed Indexes}

Violation of chargeability limits per line $\left(\mathrm{VCL}_{\mathrm{L}}\right)$ : This index is given by (1) and allows identifying those lines which are most overloaded for each scenario and under each contingency. In this case, NL, NC and NK are the number of lines, contingencies and scenarios, respectively. $\mathrm{S}_{\mathrm{l}, \mathrm{k}, \mathrm{c}}$ is the apparent power flow of line 1 , in scenario $\mathrm{k}$, under contingency $\mathrm{c}$, and $\mathrm{S}_{1}^{\max }$ is the maximum apparent power flow limit of line 1 . Note that in this case only lines over $90 \%$ of chargeability are considered in the index.

$$
\mathrm{VCL}_{\mathrm{L}}=\frac{1}{\mathrm{NL}} \sum_{\mathrm{k}=1}^{\mathrm{NK}} \sum_{\mathrm{c}=1}^{\mathrm{NC}} \max \left[\left(\mathrm{S}_{\mathrm{l}, \mathrm{k}, \mathrm{c}}-0,9 \mathrm{~S}_{\mathrm{l}}^{\mathrm{max}}\right), 0\right]
$$

Average violation of chargeability limits per operative scenario $\left(A V C L_{k}\right)$ : This index is given by (2) and permits to identify the scenarios that result in most overloads. Note that $\mathrm{AVCL}_{\mathrm{k}}$ is a vector of $\mathrm{k}$ scenarios that contains the average overloads of all lines.

$$
\mathrm{AVCL}_{\mathrm{k}}=\frac{1}{\mathrm{NC}} \frac{1}{\mathrm{NL}} \sum_{\mathrm{c}=1}^{\mathrm{NC}} \sum_{\mathrm{l}=1}^{\mathrm{NL}} \max \left[\left(\mathrm{S}_{\mathrm{l}, \mathrm{k}, \mathrm{c}}-0,9 \mathrm{~S}_{\mathrm{l}}^{\mathrm{max}}\right), 0\right]
$$

Violation of voltage limits per node $\left(\mathrm{VVL}_{\mathrm{n}}\right)$ : This index is given by (3) and allows identifying critical nodes in terms of low voltages, both in normal operation and under single contingencies over a specified set of scenarios. In this case, NB stands for number of buses, $V_{n, k, c}$ is the voltage magnitude of bus $\mathrm{n}$, under scenario $\mathrm{k}$, for contingency $\mathrm{c}$; finally $\mathrm{V}_{\mathrm{n}}^{\min }$ is the minimum voltage limit of bus $n$.

$$
\mathrm{VVL}_{\mathrm{n}}=\frac{1}{\mathrm{NB}} \sum_{\mathrm{k}=1}^{\mathrm{NK}} \sum_{\mathrm{c}=1}^{\mathrm{NC}} \min \left(\mathrm{V}_{\mathrm{n}, \mathrm{k}, \mathrm{c}}-\mathrm{V}_{\mathrm{n}}^{\min }, 0\right)
$$

Average violation of voltage limits per operative scenario $\left(\mathrm{AVVL}_{\mathrm{k}}\right)$ : This index, given by (4), allows identifying the most critical scenarios in terms of low voltages, for normal operation and under contingencies. Note that this index is analogous to $\mathrm{AVCL}_{\mathrm{k}}$, but instead of ranking chargeability, it ranks scenarios with the most critical voltage profiles.

$$
\mathrm{AVVL}_{\mathrm{k}}=\frac{1}{\mathrm{NC}} \frac{1}{\mathrm{NB}} \sum_{\mathrm{c}=1}^{\mathrm{NC}} \sum_{\mathrm{n}=1}^{\mathrm{NB}} \min \left(\mathrm{V}_{\mathrm{c}, \mathrm{n}, \mathrm{k}}-\mathrm{V}_{\mathrm{n}}^{\min }, 0\right)
$$

The main objective of the location of DG in the transmission system consists on minimizing the weighted sum of the proposed indexes as indicated in (5). In this case $w_{1}$ to $w_{4}$ are weighting factors.

Min $O F=w_{1} \mathrm{VCL}_{\mathrm{L}}+w_{2} \mathrm{AVCL}_{\mathrm{k}}+w_{3} \mathrm{VVL}_{\mathrm{n}}+w_{4} \mathrm{AVVL}_{\mathrm{k}}(5)$

\section{Metaheuristic approach}

The selected metaheuristic approach to minimize equation (5) is a conventional GA. This method is inspired on the evolution process. It starts with a population of individuals or candidate solutions. Every individual has an associated fitness or objective function. Through the generations the fittest individuals have more chances to survive and reproduce. Figure 1 depicts the representation of a candidate solution or individual of a GA. It consists on a binary vector that indicates the position of different GD units within a 6-bus power system. In this case, DG units are located at nodes 4,5 and 6. Note that conventional generation, labeled as CG is not included in the codification.

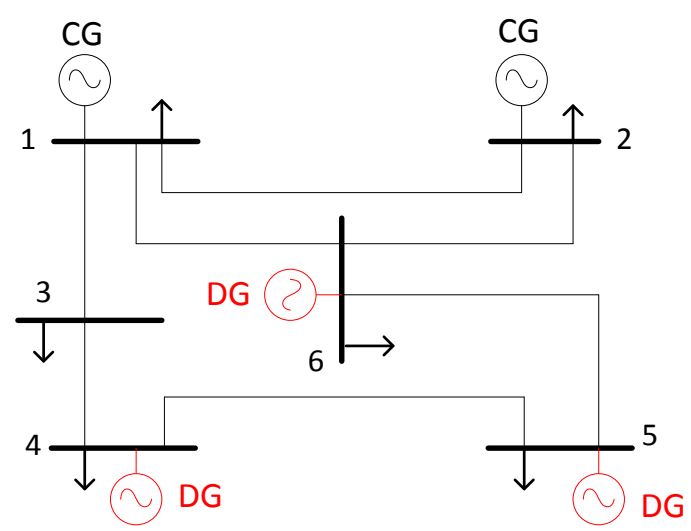

\begin{tabular}{ll|l|l|l|l|l|} 
& \multicolumn{1}{c}{1} & 2 & 3 & 4 & 5 & 6 \\
Candidate solution: & 0 & 0 & 0 & 1 & 1 & 1 \\
\cline { 2 - 7 }
\end{tabular}

Fig. 1. Codification of solutions 
The initial population is randomly generated among the system buses. Then, the fitness function, given by equation (5) is computed for each candidate solution using the software Matpower [13]. In this step, several power flows are computed considering different operative scenarios for normal conditions and under single contingencies. Once the fitness functions are computed for each individual, tournament selection is performed. In this step, a subset of $k$ individuals is selected from the current population and the one with the best objective function is chosen as the parent. The parents must them pass through the recombination stage in which they exchange information at a single position and generate new offspring. The offspring are then mutated and from the combined population of parents and offspring the best individuals are selected, keeping the size of the initial population constant. In this sept the worse individuals are discarded. The process is repeated until a pre-defined number of generations is reached. Figure 2 depicts the flowchart of the implemented GA.

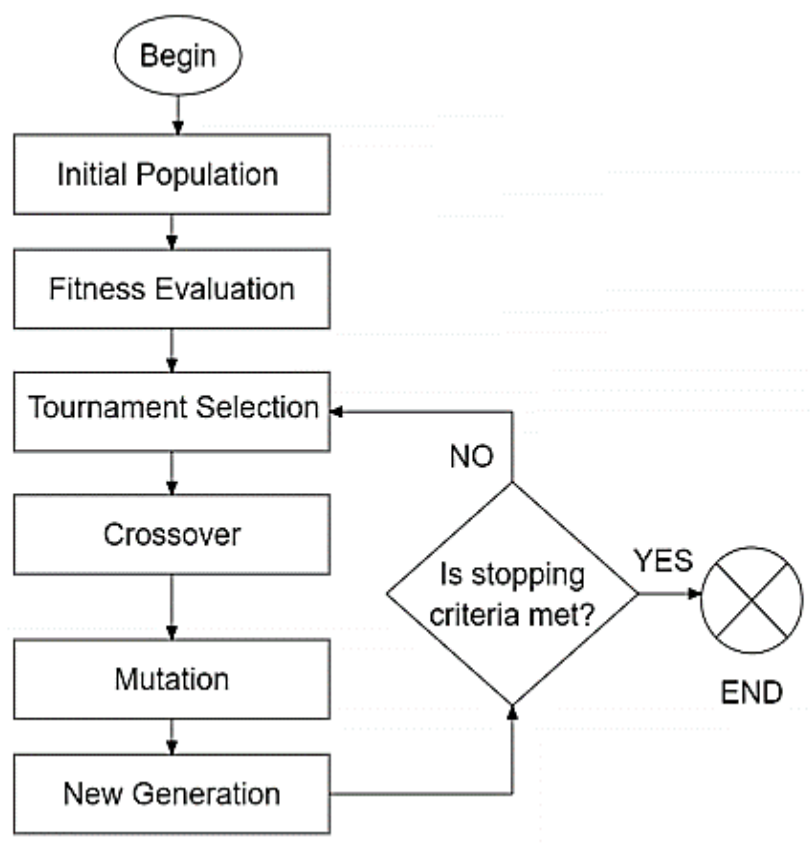

Fig. 2. Flowchart of the implemented GA.

\section{TESTS AND RESULTS}

To show the applicability of the proposed approach several tests were performed on the IEEE 30 and 57 bus test system. For both cases a population of 50 individuals with crossover and mutation rates of $80 \%$ and $20 \%$, respectively were considered. The maximum number of generations is both cases was set to 100 . Figure 3 depicts the best solution obtained with the IEEE 30 bus test system. In this case, a maximum of 5 DG units with capacity of $5 \mathrm{MW}$ each was considered. The results show that the best allocations for these units are at nodes 7, 12, 19, 21 and 30. The location of these GD units improves the fitness function in $35.5 \%$ which means that both, chargeability and voltage profile are significantly improved.

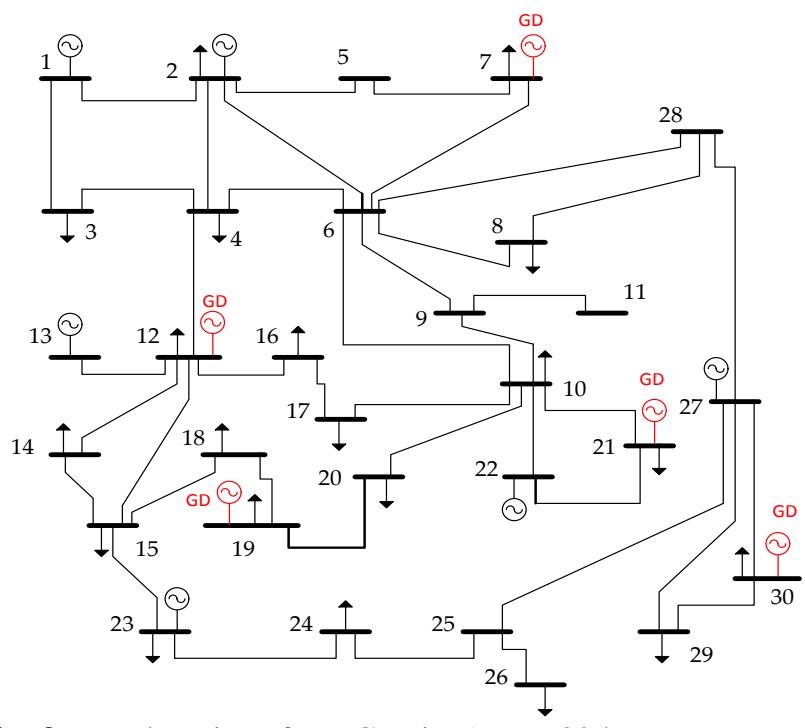

Fig. 3. Best locations for DG units (IEEE 30 bus test system)

Figure 4 depicts the results for the IEEE 57 bus test system. This system has a total demand of $1250.8 \mathrm{MW}$. In this case, a maximum of 6 DG units with capacity of $5 \mathrm{MW}$ each was considered. The best locations obtained with the proposed GA are at nodes 19, 27, 31, 32, 33 and 36. The objective function is reduced in $22.7 \%$. Nevertheless it was found that is not possible to completely eliminate all chargeability problems under single contingencies. This means that the system requires either a higher participation of DG or reinforcements in several circuits.

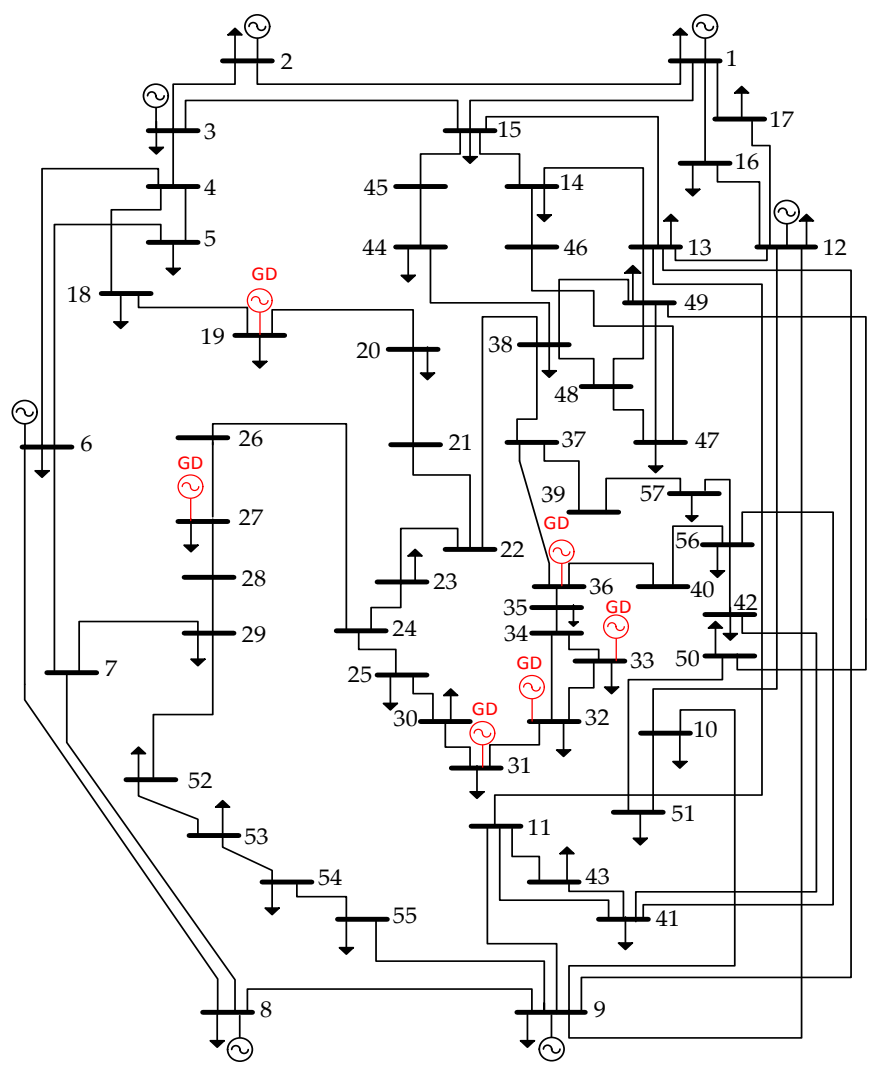

Fig 4. Best locations for DG units (IEEE 57 bus test system) 


\section{CONCLUSIONS}

In this paper a methodology for the optimal allocation of DG by means of a GA was presented. Four indexes were designed to identify critical nodes regarding violations of chargeability and voltage limits, under different operative scenarios, for normal operation and under single contingencies. The operative scenarios were generated based on the availability of centralized generation. To show the effectiveness of the proposed methodology, several tests were performed on the IEEE 30 and 57 bus test systems. In the first system it was observed that a correct location of the DG can reduce the objective function up to $35.5 \%$, reducing significantly overloads in all operative scenarios under contingencies. On the other hand, for the IEEE 57 bus test system a reduction of $22.7 \%$ of the objective function was obtained; nonetheless, it was observed that in scenarios there were still overloads under single contingencies. This means that the system requires a higher participation of DG or more system reinforcements. It is concluded from this study that the correct allocation of DG contributes to the reduction of overloads and improves voltage profile, in normal operation and under contingencies although it might not be the unique solution to solve these problems in power systems.

\section{ACKNOWLEDGEMENTS}

The authors gratefully acknowledge the support from the Colombia Scientific Program within the framework of the call Ecosistema Científico (Contract No. FP44842- 218-2018). The authors also want to acknowledge Universidad de Antioquia for its support through the project Estrategia de Sostenibilidad.

\section{REFERENCES}

[1] F. S. Abu-Mouti y M. E. El-Hawary, Optimal Distributed Generation Allocation and Sizing in Distribution Systems via Artificial Bee Colony Algorithm, IEEE Trans. Power Deliv., 26 (2011), 20902101.

[2] D. Bhowmik y A. K. Sinha, Cost-based allocation model for hybrid power system considering solar, wind and thermal generations separately, IET Gener. Transm. Amp Distrib., 11 (2017), 4576-4587.

[3] A. Al-Sabounchi, J. Gow, y M. Al-Akaidi, Simple procedure for optimal sizing and location of a single photovoltaic generator on radial distribution feeder, IET Renew. Power Gener., 8 (2014), 160-170.

[4] Y. Li et al., «Distributed Generation Grid-Connected Converter Testing Device Based on Cascaded H-Bridge Topology, IEEE Trans. Ind. Electron., 63 (2016), 21432154.

[5] X. Chen, W. Wu, y B. Zhang, Robust Restoration Method for Active Distribution Networks, IEEE Trans. Power Syst., 31 (2016), 4005-4015.
[6] H. A. Gil y G. Joos, On the Quantification of the Network Capacity Deferral Value of Distributed Generation, IEEE Trans. Power Syst., 21 (2006), 15921599.

[7] S. Liu, X. Wang, y P. X. Liu, A Stochastic Stability Enhancement Method of Grid-Connected Distributed Energy Storage Systems, IEEE Trans. Smart Grid, 8 (2017), 2062-2070.

[8] D. C. L. Tamayo, P. A. N. Burgos, y J. M. L. Lezama, Optimal Location and Sizing of Distributed Generation: A Review of the State of the Art, Rev Politecnica, 11 (2015), 99-110.

[9] A. M. El-Zonkoly, Optimal placement of multidistributed generation units including different load models using particle swarm optimisation, Transm. Distrib. IET Gener., 5 (2011), 760-771.

[10] A. Marimuthu, K. Gnanambal, y R. Priyanka, Optimal allocation and sizing of DG in a radial distribution system using whale optimization algorithm, 2017 International Conference on Innovations in Green Energy and Healthcare Technologies (IGEHT), 2017, 15.

[11] M. Junjie, W. Yulong, y L. Yang, Size and Location of Distributed Generation in Distribution System Based on Immune Algorithm, Syst. Eng. Procedia, 4 (2012), 124132.

[12] A. Casadiegos et al., Impact assessment of demand response and distributed generation in the colombian power system chargeability, 2017 IEEE PES Innovative Smart Grid Technologies Conference - Latin America (ISGT Latin America), 2017, 1-6.

[13] R. D. Zimmerman, C. E. Murillo-Sanchez, y R. J. Thomas, MATPOWER: Steady-State Operations, Planning, and Analysis Tools for Power Systems Research and Education, IEEE Trans. Power Syst., 26 (2011), 12-19. 